\title{
INVESTIR LA VILLE, ENGAGER LE CORPS : LES FLASH MOBS COMME FORMES DE MOBILISATION FUSIONNELLE ET SPECTACULAIRE DE LA JEUNESSE
}

\author{
Myriam Bahuaud et Agnès Pecolo ${ }^{1}$
}

\begin{abstract}
Dans cette contribution il s'agit de se centrer sur les « flash mobs » apparus au début des années 2000. Un flash mob est le rassemblement de courte durée d'un groupe de personnes dans un lieu public pour y effectuer une action concertée avant de se disperser rapidement. Généralement organisé au moyen d'Internet, les participants, pour la plupart, ne se connaissent pas et pourtant feront " foule » éphémère. Notre hypothèse centrale est que cette forme de mobilisation est symptomatique de nouveaux patterns de l'engagement, représentante d'un engagement "hypermoderne » animé par une génération connectée.
\end{abstract}

\section{Investir la ville, engager le corps : les flash mobs comme formes de mobilisation fusionnelle et spectaculaire de la jeunesse}

Un flash mob est le rassemblement de courte durée d'un groupe de personnes dans un lieu public pour y effectuer une action concertée (généralement une chorégraphie) avant de se disperser rapidement. Organisé au moyen d'Internet, il se déroule dans un espace-temps bien arrêté, codifié par un signal de départ et de fin. Dispersés sur le réseau, les participants ne se connaissent pas pour la plupart et convergent vers un lieu où ils capteront l'intérêt du public et délivreront un message.

1 Myriam Bahuaud et Agnès Pecolo sont Maîtres de conférences en Sciences de l'Information et de la Communication, Laboratoire MICA (Médiation, Information, Communication, Art / EA 4426), Université Bordeaux Montaigne - ISIC (Institut des Sciences de l'Information et de la Communication). Myriam Bahuaud est responsable du master «Communication et générations : étude des publics». Agnès Pecolo est directrice de l'ISIC.

Recherches en communication, $\mathrm{n}^{\circ} 42$ (2016). 
Apparus en juin 2003 à New York, et en août de la même année en France ${ }^{1}$, les flash mobs ont aujourd'hui leurs célébrités et leurs dérivés ${ }^{2}$, leurs sites, pages Facebook et comptes Twitter consacrés. Le terme flash mob devrait être dissocié de celui de smart mob, concept proposé par Howard Rheingold dès 2002, en ce qu'il n'implique pas systématiquement un engagement citoyen ou une promotion commerciale mais parfois simplement un «être ensemble » comme nous le verrons. Le concept de smart mob se réfère à de nouveaux modes d'organisation pour des individus capables d'agir de concert même sans se connaître, en utilisant des outils technologiques qui permettent une coopération au sens d'action collective (Rheingold, 2003). L'auteur voit la collision entre les mondes virtuels, sociaux et physiques comme une nouvelle forme de pouvoir social. Néanmoins, nous parlerons ici de flash mobs, quels que soient les objectifs et champs d'applications, car nous nous intéressons aux spécificités d'un mode d'expression (parmi d'autres) que les fans utilisent lors d'un hommage, les associations pour soutenir une cause, les marques pour faire participer leurs consommateurs et les organisations pour motiver leurs troupes. La coordination de publics disséminés sur le réseau qui se retrouvent le plus nombreux possible en un lieu physique pour une monstration publique est au cœur de notre développement.

Pour explorer les contours et enjeux des flash mobs, un corpus de presse généraliste et professionnelle a été étudié ${ }^{3}$ afin de cerner la diffusion de ce dispositif communicationnel dans la société. Des questionnaires passés auprès d'étudiants ont permis de saisir par ailleurs leurs connaissances et participations, opinions et représentations des flash mobs ${ }^{4}$. Existe-t-il pour eux une véritable adhésion à cette forme

1 Sur le parvis du Louvre, des inconnus ont convergé et, au signal, se sont écroulés.

2 Pillow Fight Day (bataille de polochon) ; Freeze party (participants restent figés pendant un court laps de temps); No Pants Day (usagers du métro sans pantalon); Zombie walk (participants déguisés en zombie) ; cash mob (soutien financier d'enseignes locales indépendantes).

3 Corpus de la presse professionnelle en communication (Stratégies, Stratégies Newsletter et CBNews). Recherche effectuée sur Factiva du $1^{\text {er }}$ janvier 2000 au 30 septembre 2014. 37 articles publiés de 2005 à 2012. Corpus de la presse généraliste, recherche sur Factiva du 1 1er janvier 2012 au 30 septembre 2014, 109 articles retenus.

4129 étudiants en communication -20 ans en 2012-Si nous sommes bien évidemment conscients de la non représentativité de l'échantillon (étudiants, qui plus est en communication), un certain nombre de résultats peuvent néanmoins être précisé ici. Les flash mobs apparaissent comme un mode d'expression parmi d'autres, reconnu par les jeunes interrogés (pour 122 d'entre eux). Ils les définissent de manière assez 
de mobilisation urbaine ? L'objet de cette contribution est de montrer en quoi les flash mobs sont révélateurs d'une mutation qui prête aux mobilisations contemporaines, qu'elles soient citoyennes ou plus commerciales, des caractéristiques récurrentes et spécifiques que nous proposons de mettre à jour dans les deux premières parties. Notre hypothèse centrale est que les flash mobs sont symptomatiques de « nouveaux » patterns de l'engagement et représentants, nous le verrons en dernière partie, d'une participation « postmoderne » animée par une génération hautement connectée.

\section{2. Éphémère mobilisation virale}

Se mobiliser pour une cause, promouvoir une marque, adorer une idole ou simplement faire la fête, tel est l'objet des flash mobs. Éparpillés sur le réseau, les participants convergent vers un lieu pour collaborer à un évènement créé de toutes pièces et dont ils ont auparavant appris les codes sur le Net. Les flash mobbers (dont l'anonymat et l'hétérogénéité sont souvent relevés dans nos corpus comme des caractéristiques spécifiques) font alors corps autour d'une chorégraphie, un jeu... et d'un objet de mobilisation. Leur prestation, en générant surprise et curiosité, marque le public et adresse ainsi un message.

\section{Le réseau, levier et relais d'une corporalité}

Incarnation de la puissance des réseaux et des SMS dans le cadre de manifestations et de regroupements improvisés, leviers de mobilisation et d'apprentissage des codes, les flash mobs « illustrent la capacité à mobiliser les ressources immenses d'intelligences assemblées virtuellement [...] »(Bosc, 2010, p. 3). Avec l'essor d'Internet les liens entre les réseaux et le militantisme ont été réinterrogés autour des questions de combinaisons entre un engagement en ligne et l'activisme hors ligne et de circulation de l'information militante sur le Net (Granjon, 2001 ; Cardon \& Granjon, 2003). Ici, il est spécifiquement question de l'investissement conjugué de l'espace virtuel et des places publiques. La toile devient « une outre - ville » qui émerge et peut déborder à tout

précise et en ont une perception très positive (pour presque $88 \%$ d'entre eux soit 113 étudiants sur 129) à travers les termes et expressions suivants : communion (d'une masse), communauté / rassemble (les gens) rassemblement / rencontre, faire des rencontres/ se regrouper/ réunion (du monde, des gens, des personnes), union, sentiment d'unité /proximité/cohésion / partager / dynamique de groupe, créer des liens, tisser lien social / ensemble / esprit d'équipe / participation. 
moment de ses réseaux virtuels (Boullier, 2010, p. 135). Le dispositif est toujours complété en aval par des extensions numériques (expériences prolongées), permettant de (re)passer de la rue à la toile. La technologie, relais d'amplification, permet à un territoire circonscrit de basculer en espace virtuel mondial et offre à la manifestation, systématiquement filmée, une audience démultipliée et déterritorialisée (certains étudiants interrogés de parler de " performance » en rapport au nombre de participants, à la synchronisation de la chorégraphie et à l'importance de la viralité générée). L'information propagée est estimée à hauteur de sa capacité à être partagée et détournée, reprise et commentée (Gunthert, 2013). Dans le cas des évènements arrangés par des agences de communication, on ne se retrouve plus entre inconnus mais entre professionnels organisés (danseurs, musiciens, comédiens) ce qui remet en question la notion d'horizontalité. Par ailleurs, la suspicion que la surprise du public soit feinte, que l'engouement ne soit pas (vraiment) spontané est là mais peu importe au final, du moment que le phénomène de contagion opère. Le pari est gagné dès lors que l'on fait parler de soi et ce, sur le mode sympathique (partage, communication, joie) ou/ et choquant (frayeur, angoisse précédant le soulagement comique), tel Nivéa qui fait vivre un stress redoutable à des inconnus en plein aéroport pour promouvoir l'efficacité de ses déodorants. Articulant terres urbaines et virtuelles, jouant des passerelles entre le réel et le numérique, ces dispositifs couplent rue, corps et proximité (besoin d'appropriation-enracinement), avec réseau, virtuel et propagation (besoin d'intensification-déracinement). Les représentations recueillies lors de notre enquête mettent à jour la valorisation d'un engagement double : d'une part une expression très locale liée au territoire avec le désir de se réapproprier un espace proche (fêtes de Bayonne par exemple), et d'autre part une alliance synchronisée aux quatre coins du monde grâce aux technologies (fans de Black Eyed Peas à Chicago). Déterritorialisation virtuelle et territorialisation urbaine se répondent, citoyens du monde mais aussi habitants du coin, les participants recherchent les interconnexions, être visibles et propagés dans la rue comme dans le « cyberterritoire » (Marchal \& Stébé, 2014). "A un imaginaire moderne fait de déracinement de l'individu, nos contemporains [...] opposeraient un imaginaire postmoderne fait de tentatives de ré-enracinement » (Badot \& Cova, 2003, p. 80). Le basculement du statut on line au statut on foot fait précisément la jouissance même de la participation (Boullier, 2010). Le fan virtuel se métamorphose en participant réel. 


\section{Trois petits tours et puis s'en vont}

Coup d'éclat visant à marquer les pupilles et les sens, les flash mobs, ancrés dans une culture du choc, veulent frapper les esprits par des actions aussi soudaines que brèves mais relevant néanmoins d'une organisation complexe (invitation/inscription sur un site, répétition, courriel pour lieu, date et horaire). Ces rassemblements éphémères et effervescents (Maffesoli, 2001) exploitent les clés et codes de la communication évènementielle à l'image des campagnes choc dont les organisations à buts non lucratifs se font les spécialistes (des cercueils ambulants pour la sécurité routière par exemple). Il s'agit d'être toujours plus créatif et original (critères souvent relevés par les enquêtés ${ }^{1}$ ) afin de capter l'attention du public de manière insolite et innovante, mais aussi l'intérêt des réseaux professionnels et bénéficier d'un fort relais médiatique. Les flash mobs ne peuvent pas être répertoriés tant leur nombre est élevé ${ }^{2}$ mais riment tous avec fugacité et, sauf à être particulièrement marquants ${ }^{3}$, tombent très vite dans l'oubli, noyés dans la masse et chassés par d'autres. La précarité des flash mobs se repère tant dans leur format que dans leur mémoire. Après dispersion, reste le partage sur le Net d'un "j'y étais et toi ? » qui prolongera un peu la fête. Ludiques expériences pour la plupart, l'heure semble être à l'humeur rieuse et infantile pour entretenir l'« âme d'enfant » d'adultes devenus juvéniles (Pecolo \& Bahuaud, 2014). Le jeu, et plus loin le divertissement, est pris comme un puissant outil de communication, une manière dans l'air du temps de faire connaitre une marque ou une organisation, un territoire ou une cause mais aussi de faire société. Selon Aurélien Fouillet (2014), nous assisterions à la construction de

1 Les étudiants interrogés insistent sur l'originalité, tant au niveau de la performance de groupe (aspects scénaristiques) que d'une « autre manière de $\mathrm{s}$ 'exprimer » très « tendance » (original, une autre façon de s'exprimer / peu commun / innovant/ inventif/ imaginatif / fun /tendance / impressionnant / performance de groupe).

2 Aucun article ne mentionne les flash mobs avant 2005 dans la presse professionnelle analysée alors que les publications généralistes françaises en relatent dès leur apparition en 2003. Ces publications généralistes continuent d'ailleurs de rapporter de très nombreux flash mobs organisés sur le territoire français, lors d'événements festifs, culturels et/ou associatifs, tant au niveau local que national (2 208 articles en 2013 et 1381 de janvier à septembre 2014) alors que tout discours professionnel a disparu à leur encontre dans les supports analysés depuis avril 2012.

3 Les «célébrissimes» laissent trace soit pour le nombre record de leurs participants (Black Eyes Peas à Chicago ou Michael Jackson à Mexico, tous deux en 2009), soit pour le nombre d'évènements organisés sur le thème (chorégraphies autour de Happy de Pharell Williams en 2014, des Harlem Shake en 2013 ou de Gangnam style de Psy en 2012 par exemple). 
nouvelles formes de socialité par le prisme d'un « empire ludique » qui vise à ré-enchanter le monde, en réponse, à la fois, à la fin de certains grands récits (Lyotard, 1979) et à l'émergence d'autres tels que l'utopie numérique, décrite par Fred Turner (2012) comme un héritage de la contre-culture américaine des années 1970 (liberté d'expression, quête d'authenticité créative et façonnage de communautés virtuelles).

\section{Festive mobilisation fusionnelle}

Le but ultime est la mise en corps d'une arène numérique (Monnoyer-Smith \& Wojcik, 2014), pour une métamorphose en un «être » symbiotique que seule la présence permet. Les jeunes interrogés mettent en avant « la coordination-synchronisation » du rendez-vous, l'expérience collective et les liens sociaux tissés. Sans aucune hésitation ces jeunes sont attirés par un « faire ensemble».

\section{La ville spectaculaire et participative}

Les flash mobbers transforment leur public en acteur de la promotion et les lieux publics en espaces médiatiques. Plus que simple « tendance », les flash mobs s'inscrivent dans un mouvement culturel plus large, le happening. La technologie a tout changé en permettant de multiplier les connexions et en même temps ne fait d'abord qu'appliquer à (très) grande échelle de traditionnelles pratiques participatives des décennies antérieures (les "performances » spontanées dans l'espace public des années 1960 ou les free parties des années 1990). Les flash mobs font ainsi écho à la " rhétorique participative » prégnante dans les discours communicationnels actuels (Hamel, 2014). En effet, et en lien avec les potentialités du digital et l'éclatement des frontières à l'œuvre entre marketing, médias et publics, les individus, devenus « collaborateurs » et interprètes, partenaires et co-créateurs, sont invités à co-construire l'expérience avec un « idéal d'une création partagée » (Aïm, 2012).

L'événement, insolite, attire les passants et les amène à échanger entre eux dans des espaces a priori plus co-fréquentés que relationnels. Les lieux investis comme points de contact (cités dans les articles du corpus presse - rue, place, espace commercial, aéroport, gare, sur le marché, dans le métro) sont des espaces partagés et intergénérationnels par excellence. Pour la grande majorité des étudiants interrogés, la valorisation de ces manifestations repose sur le fait qu'elles « rassemblent les gens ». L'idée de « communion et communauté, de 
rencontre et de regroupement, de réunion et d'union » est fort présente dans notre enquête de même que les notions «d'unité, de proximité, de cohésion et de partage ». Les liens sociaux (" tisser, créer ») sont mis à l'honneur dans la presse étudiée et, au-delà de l'âge varié des participants ${ }^{1}$, est relevée la mixité sociale. "La solidarité » entre des individus de « statuts sociaux », « classes sociales », différents, qui se retrouvent « tous égaux » autour d'une « activité », commune est appréciée. Se rassembler sans se ressembler tout en faisant corps commun. La rue se révèle être un véritable espace de socialisation, un terrain d'expérimentations et de mises en scène de soi exploité dans le cadre des flash mobs (Amsellem-Mainguy, 2012). Un entretien mené avec un organisateur et participant, fait ressortir qu'au-delà de simples rencontres «In Real Life » entre fans d'un même objet culturel, les flash mobs sont des moyens d'expression (« un moyen pour la communauté de se montrer et de promouvoir le produit culturel en le mettant en scène pour le public ») d'identification des fans (se compter, essayer de trouver de nouveaux fans qui partagent la même passion, de les recruter) et de construction identitaire pour le groupe participant (se conforter et se reconnaître, se définir « fédérer la communauté »). Nous retrouvons ces éléments dans l'analyse de Sandrine Lefranc et Isabelle Sommier qui montrent en quoi la mobilisation émotionnelle a une double stratégie : identitaire (à visée interne, production identitaire et socialisation militante) et de captation de soutiens (à visée externe, pression sur les cibles et exposition de la justesse de la cause) (2009). Ainsi, une mise en scène des émotions (indignation, compassion, enthousiasme) peut être force mobilisatrice. Un flash mob devient un élément amplificateur dans l'action collective voire un facteur de politisation (Cossart \& Taïeb, 2011), comme le démontre Tatyana Shunkan dans son étude de la jeunesse contestataire en Biélorussie (2008). Nouvelles formes de mobilisation prônées par les « nouveaux » militants, les flash mobs ont pour marque de fabrique la spectacularisation plus que le verbe bavard (canulars, occupations, démantèlements).

1 Extrait La voix du nord (25 avril 2013) « Les jeunes des centres sociaux, l'association des Papillons blancs de Dunkerque, les maisons de retraite des communes du Pays des Moulins, tous s'étaient donné rendez-vous pour danser ensemble. Pour aider les participants à répéter, la chorégraphe Yzabel Normand avait même prévu 2 chorégraphies : l'une assise pour les seniors, l'autre debout pour les plus jeunes. D'où ce grand mouvement intergénérationnel!» 


\section{Émotions urbaines partagées}

Ensemble de corps enracinés et en fusion, le flash mob pose une intensité émotionnelle avant d'être informationnelle. «La réintégration des émotions témoigne [...] de la volonté de réincarner l'engagement. [...] L'approche émotionnelle s'accompagne ainsi d'une réincorporation de l'action collective au sein de laquelle celle-ci est donnée à voir par ses expressions corporelles et non plus seulement discursives » (Lefranc \& Sommier, 2009, p. 274). Nous retrouvons ici ce que les théoriciens de la communication ont mis en avant dans les années 1990 à savoir la prédominance dans nos sociétés médiatiques, de la communication sur l'information (Bougnoux, 1995), de la communion sur la démonstration, de l'émotion sur la raison, de la sensation et de l'affectif sur la conviction et l'argumentation. De fait, la " réserve » urbaine (Boullier, 2010), cette convention qui consiste à ne rien laisser paraître de ses émotions, à ne pas engager d'interactions avec des inconnus, à rester immunisé vis-à-vis des surprises de la vie urbaine, est mise à mal par ces démonstrations intempestives, incitant au partage émotionnel autour d'une « ambiance affectuelle» (Maffesoli, 2011). La plupart des flash mobs utilise d'ailleurs la musique et sa densité émotionnelle ${ }^{1}$, permettant de faire lien et symbiose (Roueff, 2001). Les étudiants interrogés, tout comme la presse étudiée, retiennent ce partage fugace d'émotions et de frissons mais aussi la joie et la drôlerie, la détente et la convivialité. Le resurgissement des occasions festives montre que la « sortie de soi », l'éclatement vers l'autre, la nécessité de le toucher, le vécu commun est bien une constante anthropologique qui, après avoir été occultée durant toute la modernité, refait surface (Basilico, 2004). Ces mobilisations sont associées à de bons moments, gais et « bon enfant ». On danse, chante, se défoule. En " décoinçant» des lieux publics, les flash mobbers provoquent une rupture, réinjectent de l'improvisation et de l'aventure dans la monotonie normée de la vie quotidienne (Fouillet, 2014). « [...] quelque chose se diffuse : des joies, des colères, qui provoquent des peurs ou des enthousiasmes partagés mais qui, dans tous les cas, font circuler par surprise une monnaie jusqu'ici conservée sous le boisseau de l'urbanité-l'émotion » (Boullier, 2010 , p. 2). Un flash mob peut servir la cause ou la marque comme ne servir à rien du tout si ce n'est le besoin de s'amuser, de se frotter aux autres et de se réapproprier l'espace public. Lors d'une Zombie

1 Exemple le célèbre flash mob T-Mobile : karaoké géant organisé en 2009 à Trafalgar square (Londres) sur Hey Jude des Beatles. 
mob relatée dans le Populaire du Centre, le président de l'association organisatrice explique : « (...) Le but c'est de s'amuser et de faire les couillons, pas d'importuner les gens ou d'effrayer les enfants $»^{1}$. Les étudiants de notre enquête ont exprimé les bienfaits qu'un flash mob (effectué ou regardé) leur procure dans un quotidien « grisaille » perçu comme " morose ». Les évènements leur permettent l'« évasion » pendant quelques minutes, de « lâcher prise », d' " égayer » une journée grâce à « un petit moment de folie », « dynamique » et « entrainant », qui met un peu de baume au cœur et permet une « échappatoire » grâce à une « animation » inattendue.

\section{Renouvellement générationnel}

La société évolue, ses modalités politiques aussi. Une approche générationnelle du rapport à la citoyenneté est utile pour saisir en quoi les mutations sont toujours le fruit de plusieurs générations (héritages) mais aussi combien la jeunesse est vecteur de renouvellement. De là à faire des nouvelles générations des symboles artificiels de mutations sociales souhaitées, il n'y a qu'un pas.

\section{La jeunesse vectrice de "postmodernité "}

Force est de relever la réalité d'un jeune qui apprécie plus qu'il ne fait. Si les jeunes interrogés ont une perception très positive (pour $88 \%$ d'entre eux) des flash mobs, seuls 15\% des étudiants interrogés ont déclaré une participation et ce, principalement dans le contexte particulier des fêtes locales et du cadre scolaire. Ce résultat est d'autant plus prégnant que ces jeunes sont loin d'être représentatifs de la jeunesse et l'on sait que l'un des écueils à la «culture participative » est que les pratiques effectives sont plus l'œuvre d'une minorité d'internautes technophiles et à capital culturel conséquent (Proulx et al., 2014). Plus qu'une représentation d'une jeunesse digital addict et active en bloc, il est plus prudent de relever l'hétérogénéité des pratiques numériques et d'interroger les articulations entre pratiques off et on (compétences et centres d'intérêt préalables, trajectoires et socialisations antérieures) au cœur des motivations à l'implication (Monnoyer-Smith \& Wojcik, 2014). Pourtant, les nouveaux dispositifs communicationnels promus dans les réseaux professionnels de la communication, placent la participation au cœur de leur processus de légitimation et posent les (nouveaux)

1 Prenez garde aux morsures! Le Populaire du Centre, 6 mai 2014 
publics comme (évidemment) multicanaux, en attente d'expériences collaboratives, ludiques et innovantes et (forcément) porteurs d'autres formes d'engagement. Questionnons ce qui semble relever d'abord d'une construction idéologique : la fabrication d'un prototype de jeunesse, la fameuse génération $" Y\rangle^{1}$, « digital natives $»^{2}$, figure marketing, médiatique et managériale, jeunesse diplômée et équipée, mobilisée et mobile, urbaine et intégrée. Nous assistons à une féroce stigmatisation et instrumentalisation de cette génération, potentiel organisationnel et concept marketing plus que réalité sociologique complexe et multiple (Pecolo \& Bahuaud, 2016). Cette posture professionnelle repose sur une instrumentalisation des caractéristiques supposées de la société contemporaine (Aïm, 2012) régulièrement qualifiée de " postmoderne » et associée à des valeurs telles que la créativité et la culture du partage, l'expérientiel et le sensoriel, l'esthétisation et l'éphémère, l'émotion et le ludisme, l'expressivité et la proximité, l'horizontalité et la précarité (Lipovetsky, 1991, 2004 ; Lyotard, 1979 ; Maffesoli, 2001 ; Maffesoli \& Perrier, 2012). Une « hyper » société annoncée que la jeunesse actuelle, régulièrement rattachée à la métaphore du pouce pour sa culture numérique (Lardellier, 2006 ; Octobre, 2014 ; Serres, 2012), transcrirait dans la réalité. Reste à discuter ce concept de " postmodernité » tout comme d'ailleurs celui de " participation ». Un retweet ou un lien partagé sur Facebook est-ce une mobilisation? Peut-on s'engager dans son fauteuil, militer derrière un clavier? Signer (une pétition) ou payer (crowfunding) en ligne, actions qui ne prennent que quelques secondes, suffit-il à s'impliquer ? Au-delà de l'utopie \% dystopie classique autour des usages des TIC, il s'agit de débattre des échelles de légitimité prêtées aux modes d'engagement (degré d'investissement physique et intellectuel) mais aussi aux champs d'intervention jugés plus ou moins triviaux (commercial et industries culturelles). L'analyse des modalités du processus de participation (pourquoi et pour qui, comment et combien de temps, dans quel cadre) rend tout essai de définition " objective » complexe (Bernard, 2014).

\section{Nouvelles modalités d'engagement}

$\mathrm{Si}$ le réseau ne fait pas tout (importance des prédéterminations culturelles), les médias sociaux rendent possible néanmoins une

1 Enfants nés entre 1980 et 1994 (Strauss \& Howe, 1991)

2 Notion associée à M. Prensky (2001) Digital Natives, Digital Immigrants, site de M. Prensky, http://www.marcprensky.com/writing/prensky\%20-\%20digital $\% 20$ natives, $\% 20$ digital $\% 20$ immigrants $\% 20-\% 20$ part $1 . p d f$. 
captation de publics moins habituels, ordinairement exclus ou marginalisés dans l'espace public (Millette, 2014). Ils ont déjà largement prouvé leur contribution au débat citoyen, culturel (lectures et écritures collaboratives), à la contestation politique voire aux révoltes. «Les flash mobs sont devenus une tactique puissante dans le cadre de manifestations politiques, particulièrement dans des contextes répressifs » (Boyd \& Mitchell, 2015). Ces «[...] performances ont revêtu un caractère engagé, passant de la forme potache à celle de la mobilisation militante » (Grappin-Schmitt, 2010).

Sur les 104 étudiants interrogés n'ayant jamais participé, 64\% affirment être prêts à le faire, notamment si c'est sur un « thème qui plaît », une « idée originale », ou pour « une bonne cause », « utile », des participations « coups de cœur » qui montrent que la notion de plaisir (et non de sacrifice) devient déterminante pour eux. Nous retrouvons dans les résultats de cette enquête une forme de mobilisation d'une jeunesse qui sait donner (solidaire) mais plus fugacement et sans chaînes (individualiste), une forme plurielle, ponctuelle et moins dogmatique relevée par de nombreux auteurs (Becquet, 2011 ; Ion, 2005 ; Muxel, 2010). Un engagement distancié (intermittent et non entièrement dévoué) et pragmatique, tourné vers l'efficacité immédiate plus que vers les grandes rhétoriques révolutionnaires, est appuyé. Leurs propos dressent le profil de jeunes qui s'engagent plus volontiers dans un militantisme de terrain préférant l'horizontalité (des collectifs et des coordinations - démocratie participative) à la verticalité (des syndicats et partis politiques - démocratie représentative). Les jeunes évoqués semblent également artisans de leur engagement, vu comme une galaxie d'expériences singulières, une forme d'implication qui combine action, proximité et médias. Interrogée sur les formes de mobilisation de la jeunesse actuelle dans le magazine L'Express (2 mai 2012), Monique Dagnaud précise : « [...] les réseaux sociaux canalisent leur énergie autant qu'ils peuvent la faire exploser (les flash mobs). Ils sont généreux, pas individualistes et dotés d'une grande capacité d'indignation. Mais ils zappent très vite et sont rarement capables d'engagement à long terme ». Une fois de plus, précisons que si « la jeunesse » est posée classiquement comme le symbole des mutations en cours (renouvellement), les nouvelles modalités de l'engagement ne concernent pas que les jeunes et tous les jeunes. Anne Muxel dégage trois échelles temporelles qui permettent une meilleure compréhension des phénomènes politiques (2011). En premier lieu, une temporalité historique, autrement dit des marqueurs d'époque affectent toutes les 
classes d'âge et empêchent de prendre certaines attitudes et pratiques comme le seul apanage de la jeunesse (la défiance de la société civile à l'égard des institutions et le désenchantement est le fruit de plusieurs générations). En second lieu, une temporalité individuelle souligne l'évolution des formes et de l'intensité de la politisation au fil du cycle de vie (jeunesse contestataire, vieillesse conformiste). Enfin, une temporalité générationnelle scande le temps en fonction du renouveau et des changements sociétaux impulsés par les jeunes générations (la culture web).

Expériences relationnelles plus qu'idéologiques, les flash mobs peuvent être vus comme une illustration / symptôme de ces nouveaux patterns de l'engagement. Des armes ludiques et symboliques (exemple le succès des Zombie mobs), virtuelles (communautés virtuelles (Poissant et al., 2006)) mais aussi économiques (consommation responsable et " consumérisme politique » qui « [...] corroborerait, d'une part, le retrait des formes traditionnelles de participation politique et, d'autre part, un empowerment (une capacitation) de nature politique du consommateur » (Baygert, 2014, p. 76)).

Prenons garde pour conclure à ne pas opposer schématiquement deux types purs de militantismes, qui plus est en les associant aux couples hier-ancien-vieux-modernité / aujourd'hui-nouveau-jeunessepostmodernité. La réalité, complexe, est souvent bien plus hybride et la " nouveauté », bien moins inventive et inédite qu'occultée à certaines périodes.

Fortement valorisés par les étudiants interrogés, les flash mobs existent moins par leur participation effective que par le relais médiatique (positif) qui en est fait. Multiplication de petites agitations parties du net mais qui investissent l'espace public pris comme théâtre médiatique, les flash mobs sont des formes d'engagement à l'image d'une société éphémère et spectaculaire, médiatique et participative, ludique et fusionnelle. Ils sont, pour les plus jeunes, des modalités expressives de mobilisation et d'appropriation de l'espace urbain. « La rue ou la toile, mais aussi l'école, délimitent des conditions d'expérience politique plus spécifiques à la jeunesse [...] la rue est un espace investi avec plus de facilité que par les autres groupes d'âge » (Muxel, 2011, p. 307). Communion des corps et appréhension par le sensible d'un monde urbain, manifestations fugaces mais fusionnelles d'un être et faire ensemble, les flash mobs sont une envie/besoin de partage collectif de passions ou d'indignations, de chaleur et de convivialité, 
à hauteur de liens sociaux fragilisés. Une autre façon de faire lien et éventuellement politique. La tournure bien plus dramatique que les attentats en France ont soulevé ces derniers mois, ne fait qu'appuyer cet attachement aux rassemblements émotifs et symboliques qui se sont régulièrement manifestés. Pour faire corps et résistance.

\section{Références}

Aïm, O. (2012). Le transmédia comme remédiation de la théorie du récit. Terminal, (112), 43-55.

Amsellem-Mainguy, Y. (2012). Atlas des jeunes en France: les 15-30 ans, une génération en marche. Paris: Éditions Autrement.

Badot, O., \& Cova, B. (2003). Néo-marketing, 10 ans après: pour une théorie critique de la consommation et du marketing réenchantés. Revue Française du Marketing, (195), 79-94.

Basilico, S. (2004). Mythes, rites et tribalisation des sociétés post-modernes. Esprit critique, 6(4). Consulté à l'adresse http://www.espritcritique.fr/0604/ esp0604article08.html

Baygert, N. (2014). L'activisme numérique au regard du consumérisme politique : Pirates et Tea Partiers sous la loupe. Participations, 1(8), 75-95.

Becquet, V. (2011, novembre). L'engagement diversifié mais réel des jeunes. Constructif, (30). Consulté à l'adresse http://www.constructif.fr/bibliotheque/201111/1-engagement-diversifie-mais-reel-des-jeunes.html?item_id=3131

Bernard, F. (2014). Imaginaire, participation, engagement et empowerment. Communication \& Organisation, 1(45), 87-98.

Bosc, O. (2010). Les foules sont de retour. Mil neuf cent. Revue d'histoire intellectuelle, 1(28), 3-5.

Bougnoux, D. (1995). La communication contre l'information. Paris: Hachette.

Boullier, D. (2010). La ville événement : foules et publics urbains. Presses universitaires de France.

Boyd, A., \& Mitchell, D. O. (2015). Joyeux bordel : tactiques, principes et théories pour faire la révolution. Paris: Editions les liens qui libèrent.

Cardon, D., \& Granjon, F. (2003). Peut-on se libérer des formats médiatiques ? Le mouvement alter-mondialisation et l'Internet. Mouvements, 1(25), 67-73.

Cossart, P., \& Taïeb, E. (2011). Spectacle politique et participation. Entre médiatisation nécessaire et idéal de la citoyenneté. Sociétés \& Représentations, 1(31), 137-156.

Fouillet, A. (2014). L'empire ludique : comment le monde devient (enfin) un jeu. Paris: F. Bourin.

Granjon, F. (2001). L'internet militant: mouvement social et usages des réseaux télématiques. Rennes: Apogée.

Grappin-Schmitt, S. (2010). Flash Mob. Repères, cahier de danse, 1(25), 10.

Gunthert, A. (2013). La culture du partage ou la revanche des foules. In H. Le Crosnier, Culturenum : jeunesse, culture \& éducation dans la vague numérique (p. 163-175). Caen: C \& F Editions.

Hamel, P. (2014). Leçons de démocratie urbaine. Questions de communication, (25), 61-79. 
Ion, J. (2005). Militer aujourd'hui. Paris: Autrement.

Lardellier, P. (2006). Le pouce et la souris. Enquête sur la culture numérique des ados. Paris: Fayard.

Lefranc, S., \& Sommier, I. (2009). Conclusion. Les émotions et la sociologie des mouvements sociaux. In C. Traïni, Emotions... Mobilisation! Mobilisation! (p. 273-293). Paris: Presses de Sciences Po.

Lipovetsky, G. (1991). L'ère du vide: essais sur l'individualisme contemporain. Paris: Gallimard.

Lipovetsky, G. (2004). Les Temps hypermodernes. Paris: Grasset.

Lyotard, J.-F. (1979). La condition postmoderne: rapport sur le savoir. Paris: Editions de Minuit.

Maffesoli, M. (2001). Le temps des tribus. Le déclin de l'individualisme dans les sociétés postmodernes. Paris: La Table ronde.

Maffesoli, M. (2011). Tribalisme postmoderne. Sociétés, 2(112), 7-16.

Maffesoli, M., \& Perrier, B. (2012). L'homme postmoderne. Paris: François Bourin Editeur.

Marchal, H., \& Stébé, J.-M. (2014). La communication inhérente à la nouvelle condition urbaine. Questions de communication, (25), 7-20.

Millette, M. (2014). Contre publics et médias sociaux. In S. Proulx, J. L. Garcia, \& L. Heaton, La contribution en ligne: pratiques participatives à l'ère du capitalisme informationnel (p. 91-103). Québec: Presses Universitaires du Québec.

Monnoyer-Smith, L., \& Wojcik, S. (2014). La participation politique en ligne, vers un renouvellement des problématiques ? Participations, 1(8), 5-29.

Muxel, A. (2010). Avoir 20 ans en politique: les enfants du désenchantement. Paris: Seuil.

Muxel, A. (2011). La politique au fil de l'âge. Paris: Presses de Sciences Po.

Octobre, S. (2014). Deux pouces et des neurones. Les cultures juvéniles de l'ère médiatique à l'ère numérique. Paris: La Documentation Française.

Pecolo, A. (2016). La génération Y pour qualifier les enfants du millénaire. In Dictionnaire critique et encyclopédique des publics [en ligne].

Pecolo, A., \& Bahuaud, M. (2014). La transmission à l'heure du brouillage des âges et des générations : Visite en terrain publicitaire. Epistémé, (12), 35-56.

Poissant, L., Sénécal, M., \& Proulx, S. (2006). Communautés virtuelles : penser et agir en réseau. Presses de l'Université Laval.

Proulx, S., Garcia, J. L., \& Heaton, L. (2014). La contribution en ligne : pratiques participatives à l'ère du capitalisme informationnel. Québec: Presses Universitaires du Québec.

Rheingold, H. (2003). Smart mobs. Sociétés, 1(79), 75-87.

Roueff, O. (2001). Musiques et émotions. Terrain [En ligne], (37). Consulté à l'adresse http://terrain.revues.org/1280

Serres, M. (2012). Petite Poucette. Paris: Editions le Pommier.

Shukan, T. (2008). Le flash-mob : forme d'action privilégiée des jeunes contestataires en Biélorussie. Raisons politiques, 1(29), 9-21.

Strauss, W., \& Howe, N. (1991). Generations. The History of America's Future. NewYork: Quill William Morrow.

Turner, F. (2012). Aux sources de l'utopie numérique. De la contre-culture à la cyberculture, Stewart Brand, un homme d'influence. Caen: C \& F Editions. 